\title{
Analyse der Kolbengruppenreibung und -schmierung von Ottomotoren - Die Bedeutung des Ölabstreifrings
}

\author{
Julian Schäffer ${ }^{1}$ (D) $\cdot$ Georg Wachtmeister ${ }^{1}$ \\ Eingegangen: 10. Dezember 2020 / Angenommen: 28. Juli 2021 / Online publiziert: 26. August 2021 \\ (c) Der/die Autor(en) 2021
}

\section{Zusammenfassung}

Für die Realisierung der in Paris definierten Klimaschutzziele wird die Optimierung des Verbrennungsmotors weiterhin eine tragende Rolle spielen. Sowohl für den Kleinmotor im Hybridantrieb als auch für den Großmotor in Nutzfahrzeugen und Baumaschinen hat eine Effizienzsteigerung höchste Priorität. Maßnahmen zur Reduktion der mechanischen Verluste werden typischerweise mit geschleppten Reibleistungsmessungen am Vollmotor (Strip-down Analyse) bewertet. Die Ergebnisse zeigen, dass bis zu 50\% der Gesamtverluste im tribologischen System Kolben/Zylinderlaufbahn entstehen. Zur Analyse der Kolbengruppenreibung wird eine kurbelwinkelbasierte Reibkraftmessung am Einzylindermotor eingesetzt, aus der sich der Reibmitteldruck als Maß der Effizienz ableiten lässt. Mit dem sogenannten Floating-Liner Motor wird zunächst bewertet, welche Wirkung mit der Tangentialkraftreduktion des Ölabstreifrings hinsichtlich Reibungsverluste erzielt werden kann. Da sich Reibung stets im Spannungsfeld mit Ölverbrauch befindet, wird zudem die Wirkung der reduzierten Tangentialkraft auf den Ölhaushalt der Kolbengruppe analysiert. Hierzu wurde ein Transparent-Aggregat entwickelt, das die Visualisierung der Schmierölströmung als auch eine Bewertung der Ölschichtdicken ermöglicht. Mit diesem kombinierten Ansatz können Maßnahmen gefunden werden, die zu einer Reduktion des $\mathrm{CO}_{2}$-Ausstoßes bei gleichbleibenden Ölemissionen führen. Letzteres gewinnt mit dem zukünftigen Einsatz synthetisch hergestellter Kraftstoffe (eFuels) zunehmend an Bedeutung, da diese nahezu partikelfrei verbrennen und somit der Öleintrag in den Brennraum als einzige Partikelquelle verbleibt.

Availability of data and material The authors declare that all data and materials support their published claims and comply with field standards.

\section{Code availability Not applicable}

Julian Schäffer

Schaeffer@1vk.mw.tum.de
Georg Wachtmeister

Wachtmeister@lvk.mw.tum.de

1 Lehrstuhl für Verbrennungskraftmaschinen (LVK), Fakultät für Maschinenwesen, TU München, Boltzmannstraße 15, 85748 Garching b. München, Deutschland 


\title{
Piston assembly friction and lubrication analysis of gasoline engines-The influence of the oil control ring
}

\begin{abstract}
The optimization of the internal combustion engine will continue to play a key role in achieving the climate protection targets defined in Paris. Increasing efficiency has top priority both for small engines in hybrid drives and for large engines in commercial vehicles and construction machinery. Measures to reduce mechanical losses are typically evaluated with dragged friction measurements on the full engine (strip-down analysis). The results show that up to $50 \%$ of the total friction losses occur in the piston/cylinder liner tribological system. To analyze the piston assembly friction, a crank angle-based friction force measurement on the single-cylinder engine is used, from which the friction mean effective pressure can be derived as a measure of efficiency. The so-called floating liner engine is first used to assess the friction potential of tangential force reduction of the oil control ring. Since there is always a trade-off between friction and oil consumption, the effect of the reduced tangential force on the oil distribution within the piston assembly is also analyzed. For this purpose, a transparent engine was developed at the institute, which enables the visualization of the lubricating oil flow as well as an evaluation of the oil layer thickness. This combined approach enables a reduction of $\mathrm{CO}_{2}$ emissions with low oil consumption at the same time. The latter is becoming increasingly important with the future use of synthetically produced fuels (eFuels). Since the combustion of these types of fuel is almost free of particles, the oil entering the combustion chamber remains the only source of particle emissions.
\end{abstract}

\section{Einleitung}

Elektrifizierte Antriebsstränge haben in den letzten Jahren zunehmend an Bedeutung gewonnen. Gerade im letzten Jahrzehnt konnte durch zunehmende Elektrifizierung ein wesentlicher Beitrag zur $\mathrm{CO}_{2}$-Reduktion des Automobilsektors erreicht werden. Durch den starken Fokus von Forschung und Entwicklung auf Batterieelektrische Fahrzeuge (BEVs) wurde zugleich die Weiterentwicklung des Verbrennungsmotors vernachlässigt [1]. Mit der neuen Zertifizierungskategorie „Low Fuel Consumption Vehicles (LFCVs)“ versucht China diesem Trend entgegenzuwirken. Diese Kategorie umfasst Fahrzeuge mit Verbrennungsmotor, deren Kraftstoffverbrauch unter den gesetzlich definierten Zielwerten liegt. Für die Erreichung der Zielwerte ist die Entwicklung neuer Technologien zur Wirkungsgradsteigerung und Emissionsreduktion erforderlich.

Um den Anforderungen der LFCVs zu genügen, ist neben der Reduktion thermodynamischer Verluste eine Minimierung der mechanischen Verluste unabdingbar. Bei modernen Verbrennungsmotoren geht über ein Zehntel der eingebrachten Kraftstoffenergie zufolge der innermotorischen Reibung verloren, wobei der Großteil dieser Verluste dem tribologischen System Kolben/Zylinderlaufbahn zugemessen werden kann [2]. Eine Möglichkeit der Effizienzsteigerung besteht in der Reduktion der Vorspannkraft des Ölabstreifrings. Dadurch können sowohl die Mischreibungsanteile aufgrund einsetzender Dünnfilmschmierung in den Totpunkten als auch die hydrodynamischen Reibungsverluste bei hohen Relativgeschwindigkeiten gesenkt werden. Ein Eingriff dieser Form kann jedoch zu einer erhöhten Ö1durchlässigkeit des Ringfeldes führen und somit zu einem steigenden Ölverbrauch beitragen. Einzelne Öltröpfchen im
Brennraum können als Zündherde dienen und insbesondere bei den aktuellen Downsizing-Konzepten mit Turboaufladung Vorentflammungen auslösen. Des Weiteren wirken sich unverbrannte Öltröpfchen negativ auf die Lebensdauer von Abgasnachbehandlungskomponenten aus, wohingegen die verbrannten Bestandteile die innermotorische Partikelentstehung fördern [3]. Den bestehenden Zielkonflikt zwischen Reibungsverlusten und Ölverbrauch gilt es zu lösen. Hierfür werden in dieser Studie sowohl die Reibkraft als auch die Ölströmung der Kolbengruppe analysiert. Die Untersuchungen finden an einem Einzylinder-Aggregat mit optischem Zugang statt, welches die Visualisierung der Ölströmung auf der Druckseite der Kolbengruppe ermöglicht.

\section{Theoretische Grundlagen}

\subsection{Reibung der Kolbengruppe}

Die Hubkolben moderner Pkw-Ottomotoren sind typischerweise mit drei Kolbenringen ausgestattet (vgl. Abb. 3). Dabei haben die Kompressionsringe (hier: Top Ring und Nasenminutenring) primär die Aufgabe, den Brennraum zum Kurbelgehäuse hin abzudichten. Neben der Abdichtfunktion kann der als Nasenminutenring ausgeführte zweite Kolbenring zusätzlich Öl von der Zylinderlaufbahn abstreifen. Der unterste Ring wird Ölabstreifring genannt und ist für die Verteilung des zugeführten Schmieröls innerhalb des Ringfelds zuständig. Da die Kolbenringe statisch vorgespannt sind und die Kompressionsringe im Betrieb zudem mit Gasdruck beaufschlagt werden, werden die Ringlaufflächen gegen die Zylinderlaufbahn gepresst. Die entstehende Flächenpressung $p$ der Kontaktflächen, die Relativ- 


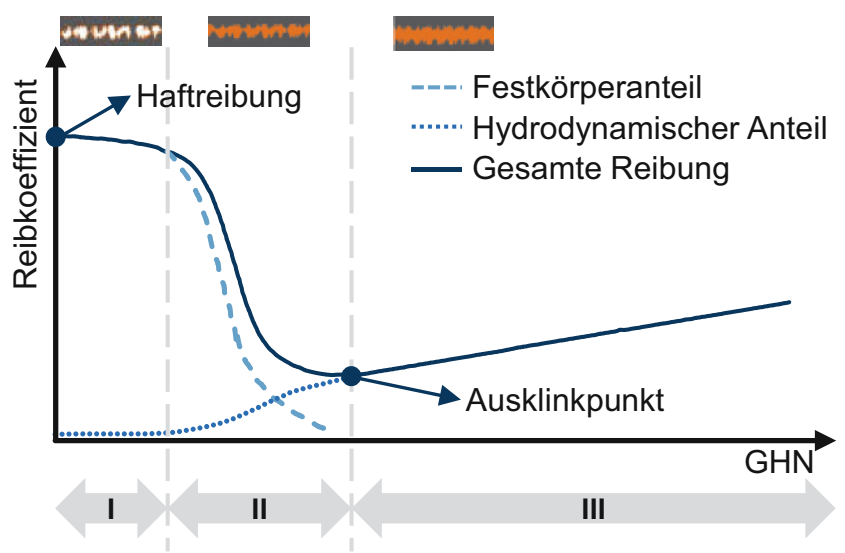

I Grenzschmierung

II Dünnfilmschmierung

III Flüssigkeitsschmierung

- Hydrodynamische Schmierung

- Elastohydrodynamische Schmierung

Abb. 1 Stribeck-Kurve, modifiziert nach [4]

geschwindigkeit $U$ der Gleitpartner und die dynamische Viskosität $\eta$ des Schmiermittels bestimmen letztendlich die Schmierfilmdicke zwischen den Kolbengruppenkomponenten und der Zylinderlaufbahn. Da sich die genannten Größen im Verbrennungsmotor kontinuierlich ändern, werden sämtliche Schmierungszustände durchlaufen, die der Abb. 1 entnommen werden können. Die dargestellte Stribeck-Kurve beschreibt den Reibungsverlauf als Funktion des dimensionslosen Schmierungsparameters GHN (Gümbel-Hersey number):

$G H N=\frac{\eta \cdot U}{p}$

Die Schmierfilmdicke weist den gleichen Trend auf wie der hydrodynamische Anteil der Stribeck-Kurve, d.h. mit zunehmender Gleitgeschwindigkeit wird der Schmierfilm dicker und mit zunehmender Pressung wird der Film dünner.

Bei der Flüssigkeitsschmierung sind die Gleitpartner durch einen ausreichend dicken Schmierfilm voneinander getrennt. Der zwischen den Kontaktflächen wirkende Druck wird gänzlich vom Schmierfilm aufgenommen. Im betrachteten System tritt dieser Schmierungszustand insbesondere an den Ringstegen auf, weshalb diese kaum verschleißen. Zwischen den Kolbenringlaufflächen und der Zylinderlaufbahn ist die Pressung deutlich höher, weshalb hier der Ölfilm sehr dünn ist. Abhängig von der Tangentialkraft, des Druckniveaus in den Ringnuten und der Gleitgeschwindigkeit kann hier der Übergang in die Dünnfilmschmierung oder gar Grenzschmierung erfolgen. Grenzschmierung bezeichnet einen kritischen Zustand, bei dem die Ölfilmdicke kleiner als die Oberflächenrauigkeit ist. Demnach geht der wirkende Druck nicht mehr in den

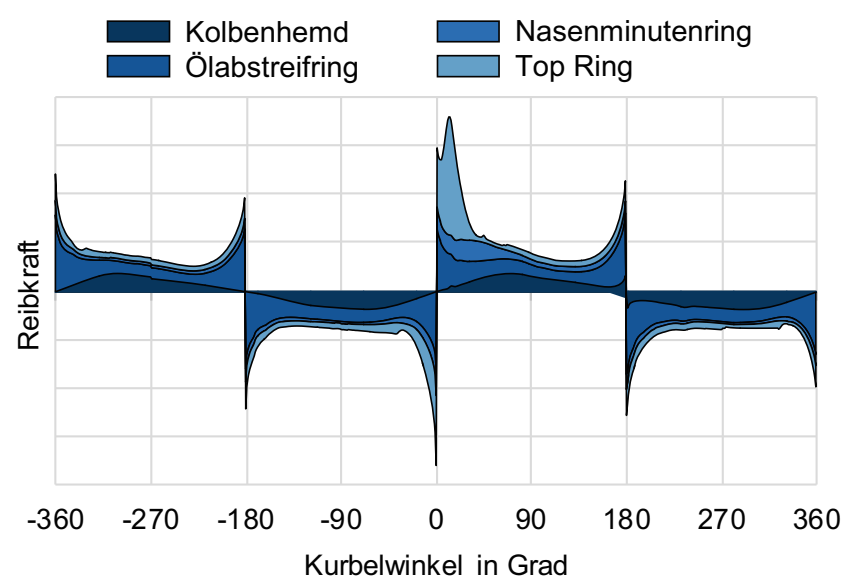

Abb. 2 Schematischer Reibkraftverlauf, modifiziert nach [5]

Schmierfilm ein, sondern direkt in die Kontaktflächen, was $\mathrm{zu}$ hoher mechanischer und thermischer Belastung führt.

Abb. 2 zeigt einen typischen Reibkraftverlauf für einen Motorzyklus. Aufgrund des hohen Hydrodynamik-anteils hat die Kolbenhemdreibung ein Maximum im Punkt der höchsten Gleitgeschwindigkeit, auf halber Hubhöhe. Der Ölabstreifring hat durch den hohen Mischreibungsanteil und die maßgebliche Belastung durch die Ringvorspannung ein sehr konstantes Verhalten über das gesamte Arbeitsspiel. In den Totpunkten kommt es zusätzlich zu einem Anstieg durch die Zunahme der Grenzschmierung (vgl. Abb. 1). Der Nasenminutenring weist eine niedrige Ringvorspannung und eine geringe Beanspruchung durch den Zylinderdruck auf, weshalb dessen Reibkraft über den gesamten Motorzyklus relativ gering ist. Die maßgebliche Beanspruchung des Top Ringes geschieht durch den Zylinderdruck während Kompression und Verbrennung. Die geringe Ringvorspannung führt in den restlichen Hubbereichen zu niedrigen Reibwerten.

Für einen bestimmten Kurbelwinkel $\varphi$ ist die Reibleistung $P_{R}$ der Kolbengruppe definiert durch das Produkt aus Reibkraft $F_{R}$ und der Relativgeschwindigkeit $U$ zwischen den Reibpartnern:

$P_{R}(\varphi)=F_{R}(\varphi) \cdot U(\varphi)$

Für die Bewertung der Effizienzsteigerung wird der Reibmitteldruck $p_{m r}$ herangezogen, der die auf das Hubvolumen $V_{H}$ normierte Reibarbeit $W_{R}$ beschreibt:

$p_{m r}=\frac{W_{R}}{V_{H}}=\frac{2 \cdot \overline{P_{R}(\varphi)}}{V_{H} \cdot i}$

Mit dem Parameter $i$ wird die Anzahl der Arbeitsspiele pro Umdrehung berücksichtigt. Für den 4-Takt Betrieb gilt somit: $i=0,5$. 


\section{2 Ölhaushalt der Kolbengruppe}

Das Schmieröl kann entlang zwei verschiedener Pfade in den Bereich zwischen Kolbengruppe und Zylinderlaufbahn eindringen. Zum einen schleudern die Wangen der Kurbelwelle und das Pleuel während der Drehbewegung Öl in Form kleiner Tröpfchen nach oben. Zusätzlich befindet sich am unteren Ende der Laufbuchse eine Kühldüse, die das Schmieröl ebenfalls nach oben in Richtung Kolben spritzt. Je nach Kolbenposition benetzt das Spritzöl entweder die Lauffläche und/oder den Innenraum des Kolbens. Letzteres dient primär der Kolbenkühlung, weniger der Schmierung. Jedoch kann ein Teil des Öls über die Ölablaufbohrungen in der Ölringnut zum Ölabstreifring strömen (vgl. Abb. 3). Das auf der Lauffläche haftende Öl gelangt in die Kolbengruppe, während sich diese in Richtung unterem Totpunkt bewegt. Dabei verbleibt ein Großteil der Ölmenge unterhalb des Ölabstreifrings. An dieser Stelle weist das Kolbenhemd eine Fase mit anschließend reduziertem Kolbendurchmesser auf, so dass sich ein Ölreservoir bilden kann. Ausgehend von diesem Reservoir kann das Öl grundsätzlich entlang drei verschiedener Pfade zum Feuersteg fließen: durch den Kontakt Kolbenring/Zylinderlaufbahn, durch die Kolbenringnuten und durch die Kolbenringstöße. Bei diesen Pfaden handelt es sich um Lücken, die das dynamisch abdichtende System aufweist.

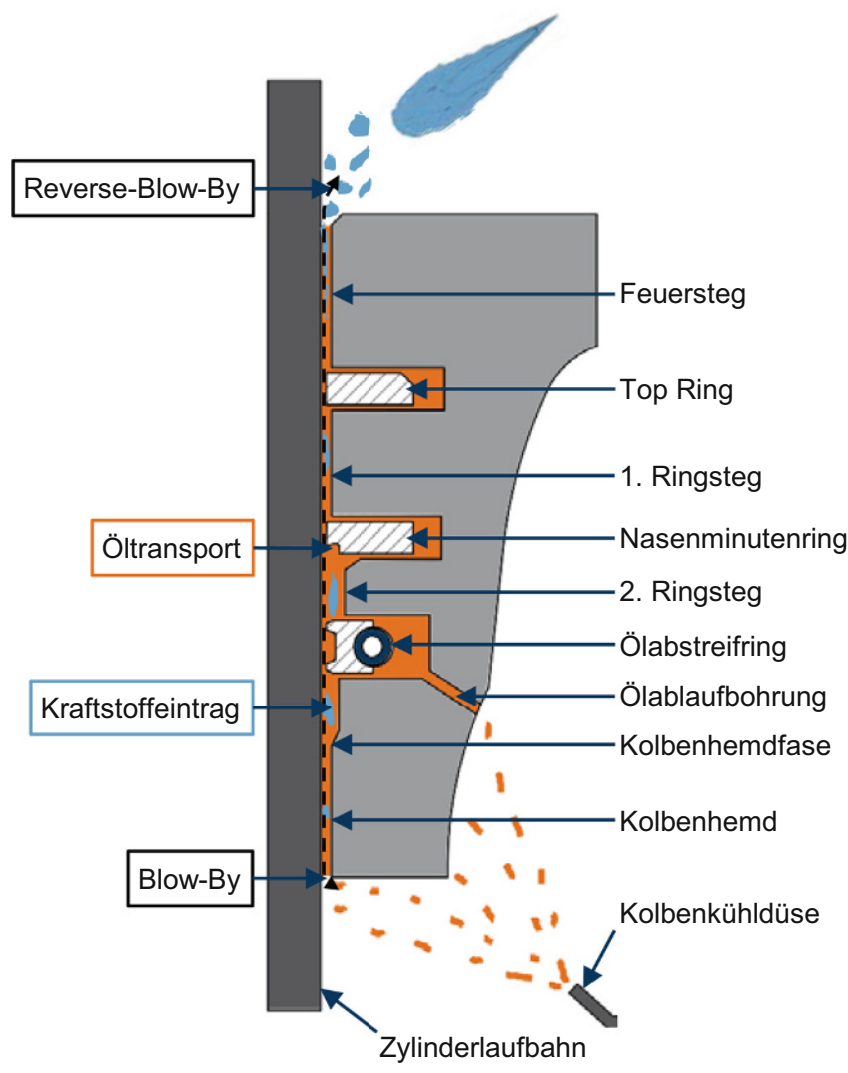

Abb. 3 Effekte in der Kolbengruppe
Wie in Abb. 3 gezeigt können Brennraumgase das Ringfeld überwinden und in Richtung Kurbelgehäuse strömen (Blow-By). Durch Scherkräfte an der Öl/Gas Grenzschicht wird ein Teil des Schmieröls in Richtung der Gasströmung transportiert. Je nach Zylinderdruckniveau kann auch eine Gasströmung in entgegengesetzte Richtung auftreten (Reverse-Blow-By). Auf diese Weise kann beispielweise zu viel Öl auf den Feuersteg gelangen, was den Ölverbrauch steigen lässt. Neben diesem Öltransportmechanismus haben die auf den Schmierfilm und auf die Komponenten wirkenden Massenkräfte einen wesentlichen Einfluss auf die Ölströmung durch das Ringfeld. Gasdruck und Massenkräfte hängen stark vom gewählten Betriebspunkt, also von Drehzahl und Last ab, weshalb das Ringpaket unter verschiedenen Randbedingungen funktionieren muss.

Die Tangentialkraft des Ölabstreifrings beeinflusst hierbei maßgeblich die auf der Laufbahn zurückbleibende Ölmenge. Das an der Zylinderwand haftende Öl kann anschließend die oberen Regionen der Kolbengruppe erreichen und folglich den Öleintrag in den Brennraum fördern.

\section{Messmethoden}

\subsection{Floating-Liner Reibungsmotor}

Für die Messung der Reibkraft zwischen Kolbengruppe und Zylinderlaufbahn wird ein Einzylinder-Ottomotor verwendet, der auf einem 2.01 Serienmotor basiert. Die Eigenschaften des Einzylinder-Aggregats können Tab. 1 entnommen werden. Die Messmethode basiert auf dem Floating-Liner Prinzip, das erstmals von Furuhama et al. [6] publiziert wurde. Mit dieser Methode ist es möglich, die Reibkraft kurbelwinkelaufgelöst zu erfassen.

Basierend auf dem System aus [6] hat Werner einen Einzylinder-Motor entwickelt [7]. Der Aufbau hat sich im Rahmen mehrerer Reibungsanalysen bewährt [8-14] und wurde auch für diese Studie eingesetzt. Gemäß Abb. 4 stellt die Floating-Liner Gruppe, bestehend aus der Zylinderlaufbuchse und dem Wassermantel, die zentrale Einheit dar.

Tab. 1 Eigenschaften des Einzylinder-Aggregats

\begin{tabular}{lll}
\hline & Serienmotor & Einzylinder \\
\hline Hubvolumen [L] & 2,0 & 0,5 \\
Hub [mm] & 92,8 & \\
Bohrung [mm] & 82,5 & \\
Pleuellänge [mm] & 144 & \\
Verhältnis r/l [-] & 0,32 & \\
Verdichtungsverhältnis [-] & $9,6: 1$ & \\
Max. Drehzahl [min ${ }^{-1}$ ] & 6800 & 5000 \\
Max. Zylinderdruck [bar] & 110 & 90 \\
Max. ind. Mitteldruck [bar] & 22 & 18 \\
\hline
\end{tabular}




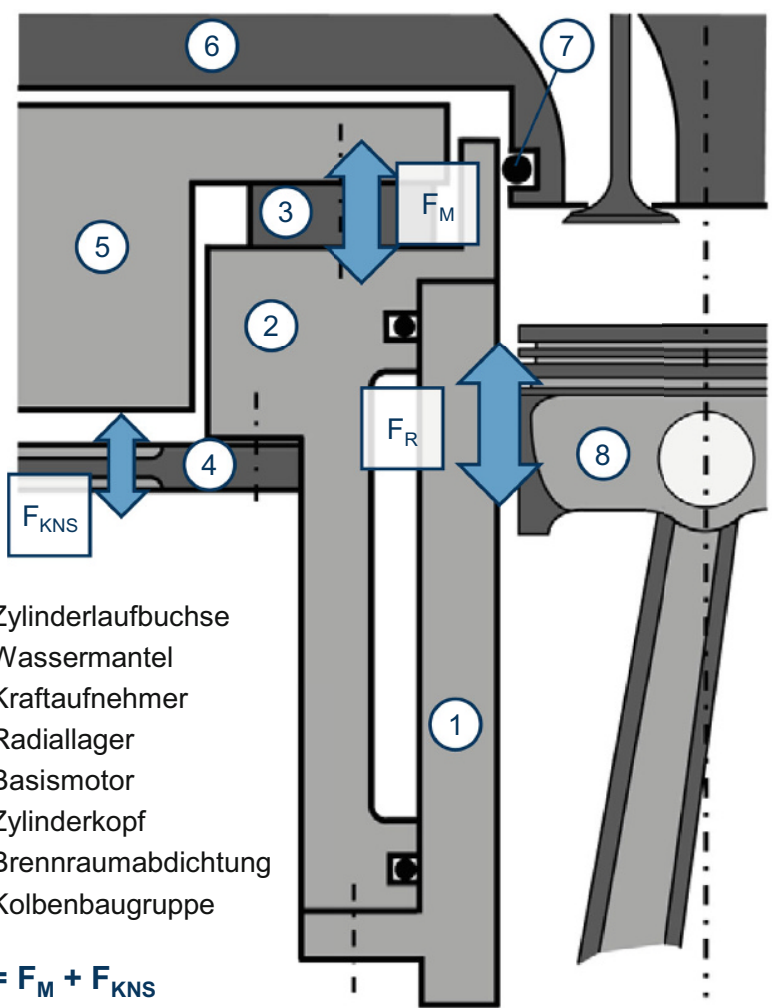

Abb. 4 Prinzip Floating-Liner Methode, modifiziert nach [14]

Diese Gruppe ist mit dem Grundmotor über zwei piezoelektrische Kraftaufnehmer (Typ: Kistler 9061A) verbunden. Durch Vorspannung der Kraftaufnehmer ist die Messung von Zug- und Druckkräften möglich. Die Abstützung der Kolbenseitenkräfte erfolgt mittels Radiallager, die sich zusammen mit den Sensorschrauben im Kraftnebenschluss der Kraftsensoren befinden. Dieser Kraftanteil verhält sich linear zur gemessenen Kraft, wodurch er statistisch ermittelt und in Form eines Kraftnebenschlussanteils $F_{K N S}$ berücksichtigt werden kann. Eine Besonderheit stellt die Abdichtung des Brennraums dar, die eine vertikale Bewegung der Floating-Liner Gruppe zulässt. Durch Anpassung der Zylinderkopfgeometrie kann die Abdichtung gemäß Abb. 4 mit einem hochtemperaturbeständigen Dichtring erfolgen. Dabei ist der Spalt zwischen Wassermantel und Zylinderkopf sehr eng gewählt, um den Ring vor den Verbrennungsgasen zu schützen. Mit diesem Konzept kann auf eine aufwändige Gasdruckkompensation sowie auf geometrische Anpassungen der Kolbenkrone nach $[6,15,16]$ verzichtet werden.

\subsection{Laser-induzierte Fluoreszenz}

Die Messtechnik zur Visualisierung der Ölschicht zwischen Kolbengruppe und Zylinderlauffläche basiert auf der Methode der laserinduzierten Fluoreszenz (LIF). Dabei wird das Motoröl mit einem speziellen Farbstoff markiert, der nach Anregung durch monochromatisches Laserlicht fluo-

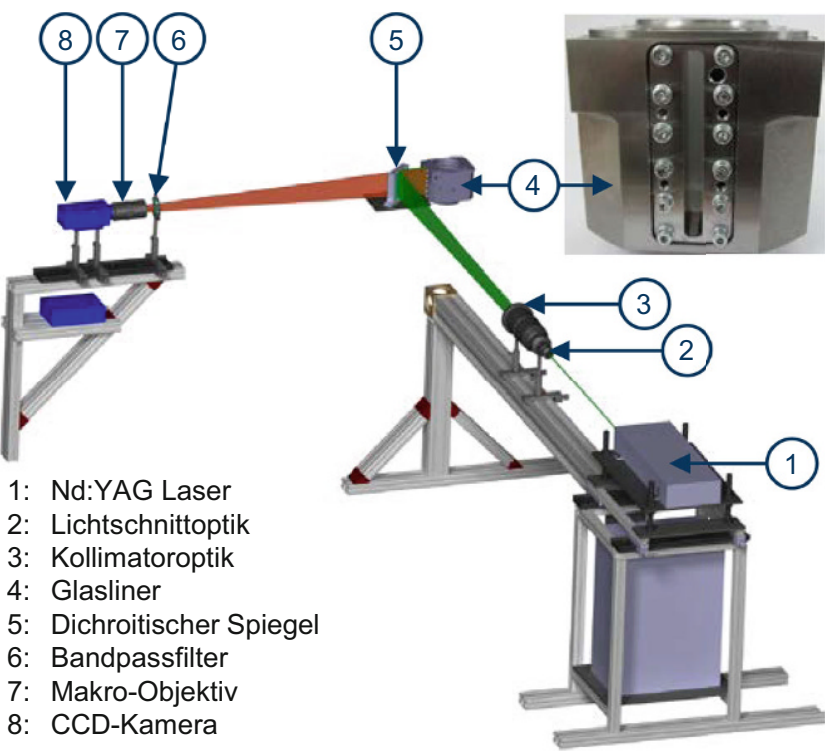

Abb. 5 Optischer Strahlengang der LIF-Messtechnik, modifiziert nach [21]

resziert. Der optische Zugang wird hierbei durch ein in das Zylindergehäuse eingesetztes Saphirfenster erreicht, wie in Abb. 5 oben rechts gezeigt. Dadurch kann die Ölschicht auf der Druckseite über den gesamten Motorzyklus detektiert werden. Das optisch zugängliche Zylindergehäuse und die Messtechnik wurden auf Basis der Arbeiten von Thirouard et al. [17, 18] und Wigger et al. [19, 20] entwickelt. Konstruktive Merkmale und Details zur Messtechnik können [21-23] entnommen werden.

Gemäß Abb. 5 dient als Anregungsquelle ein doppelt gepulster Nd:YAG-Laser, der ein monochromatisches Licht mit einer Wellenlänge von $532 \mathrm{~nm}$ aussendet. Lichtschnittund Kollimatoroptik enthalten ein Linsensystem, welches den punktförmigen Laserstrahl auf die gewünschte Fläche von $100 \times 10 \mathrm{~mm}$ aufweitet. Ein speziell beschichteter, dichroitischer Spiegel reflektiert das Laserlicht hin zur Messstelle. Die Farbstoffmoleküle des mit Rhodamin 640 und Pyrromethen 567 versetzten Schmieröls absorbieren die einfallenden Lichtphotonen, was zu einer Anregung der Moleküle in einen höheren energetischen Zustand führt. Bei der Fluoreszenz emittieren die Moleküle während der Rückkehr in den Grundzustand ein Photon, das verglichen mit dem einfallenden Laserlicht rotverschoben ist (Stokesshift). Die rotverschobenen Fluoreszenzphotonen passieren den dichroitischen Spiegel und werden von der CCD-Kamera detektiert. Dabei ist die Photonenzahl proportional zur Schmierölschichtdicke. Mit dem Bandpassfilter wird verhindert, dass Anregungslicht und Streulicht zum Detektor gelangen.

In Abb. 6 ist eine Einzelaufnahme auf der Druckseite im geschleppten Motorbetrieb bei $60{ }^{\circ} \mathrm{KW}$ nach ZOT dargestellt. Um einen quasi-stationären Zustand zu errei- 


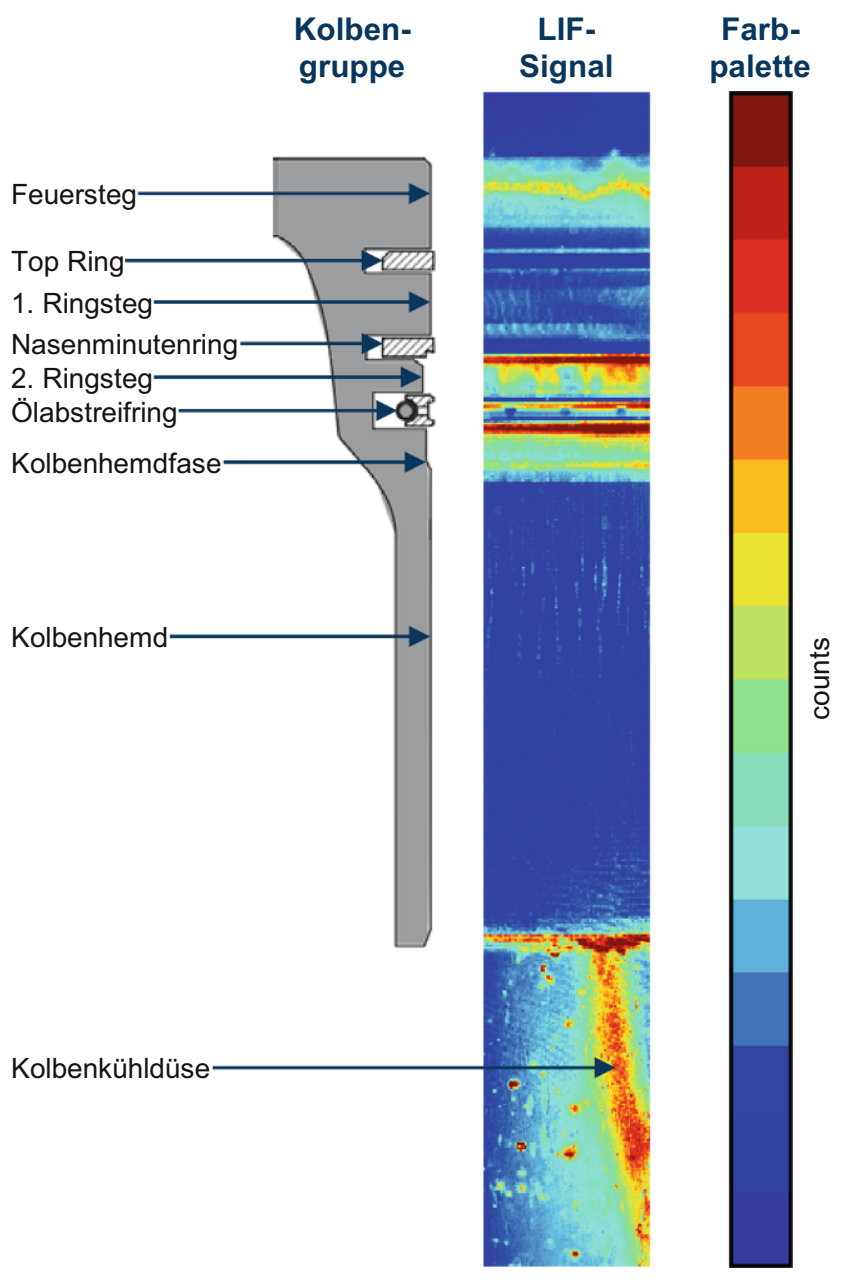

Abb. 6 LIF-Signal während der Expansion (links: Kolbengruppe schematisch; mittig: LIF-Signal; rechts: Farbpalette)

chen, wurde der Betriebspunkt für fünf Minuten gehalten. Am rechten Bildrand befindet sich die zugehörige Farbskala. Diese ist so gewählt, dass geringe Ölschichtdicken in einem dunklen Blau erscheinen und Bereiche mit dicker Ölschicht in Rot. Eine Unterscheidung der einzelnen Kolbengruppenkomponenten und -bereiche ist anhand des LIFSignals möglich. Beginnend von oben stellt der erste helle Bereich der Aufnahme den Feuersteg dar. Hier ist zu erwähnen, dass das Ölangebot auf dem Feuersteg sehr stark von dem Betriebsmodus und dem Betriebspunkt abhängt, was im Ergebnisteil deutlich wird. Zwischen Top Ring und Zylinderlaufbahn ist die Ölschicht sehr dünn, was der dunkelblaue Streifen im LIF-Signal verdeutlicht. Lediglich am Übergang von Ring zu Feuersteg ist eine starke Intensitätssteigerung zu sehen, was darauf zurückgeführt werden kann, dass durch Relativbewegung Schmieröl aus der Kolbenringnut gequetscht wird. Der 1. Ringsteg ist anhand des helleren Bereiches zwischen den beiden Kompressionsringen zu erkennen. In diesem Fall zeigt sich ein leicht verästeltes Muster, was auf eindringende Brennraumgase zurückgeführt werden kann. Unterhalb des Nasenminutenrings steigt das Ölangebot signifikant an. Hier zeigt sich die Funktion des Nasenminutenrings, der bei der Abwärtsbewegung das Öl von der Zylinderlauffläche abstreift. Damit sich das Öl auf dem 2. Ringsteg sammeln kann, ist der Kolbendurchmesser in diesem Bereich reduziert. Der Ölabstreifring ist deutlich anhand der Ablaufbohrungen im Ringkörper zu erkennen. Das vom unteren Laufsteg abgestreifte Öl sammelt sich an der unteren Ringflanke im Bereich der hierfür vorgesehenen Übergangsfase zum Kolbenhemd. Da das Kolbenhemd den größten Kolbendurchmesser besitzt und zudem grafitbeschichtet ist, ist die erfasste Fluoreszenzintensität sehr gering.

Eine absolute Messung der Ölschichtdicke kann mit Hilfe einer geeigneten Kalibrierung erfolgen. Die angewendete Kalibriermethodik berücksichtigt sowohl den Einfluss der Öltemperatur auf die Fluoreszenzintensität als auch die unterschiedlichen Reflektivitäten der verschiedenen Werkstoffe der Kolbengruppe. Details hierzu können [21, 22] entnommen werden.

\section{Ergebnisse}

Die folgende Studie ist in zwei Messblöcke unterteilt. Im ersten Block findet eine Reibungsmessung am Floating-Liner System statt, die in einer Messkampagne aus [11] durchgeführt wurde. Dabei wird die Tangentialkraft des Ölabstreifrings variiert und das Potenzial zur Reibungsreduktion bewertet. Anschließend wird eine Variation der Tangentialkraft am Transparent-Aggregat durchgeführt. Für verschiedene Betriebspunkte und Betriebsmodi wird der Einfluss auf die Ölströmung und den Ölverbrauch analysiert. Für alle Versuche wurde ein Motoröl mit der SAE Viskositätsklasse 5W30 verwendet.

\subsection{Wirkung der reduzierten Tangentialkraft auf Reibung}

In Abb. 7 ist eine ausgewählte Floating-Liner Messung dargestellt, aufgezeichnet bei $2000 \mathrm{~min}^{-1}$ und 6 bar indiziertem Mitteldruck $p_{m i}$. Das untere Diagramm zeigt den Reibkraftverlauf, das mittlere die Reibkraftdifferenz und das obere den zugehörigen Zylinderdruckverlauf.

Anhand der Reibkraftverläufe ist deutlich zu erkennen, dass die reduzierte Vorspannung des Ölabstreifrings signifikante Vorteile nahezu im gesamten Motorzyklus zeigt. Da der Ölabstreifring, anders als die Kompressionsringe, kaum vom Brennraumdruck beeinflusst wird und die maßgebliche Belastung durch die Ringvorspannung erfolgt, ist dessen Reibverhalten sehr konstant über das gesamte Arbeitsspiel. Dies erklärt, weshalb die Reduktion der Tangentialkraft sowohl im Mischreibungsbereich in den Totpunkten als auch 


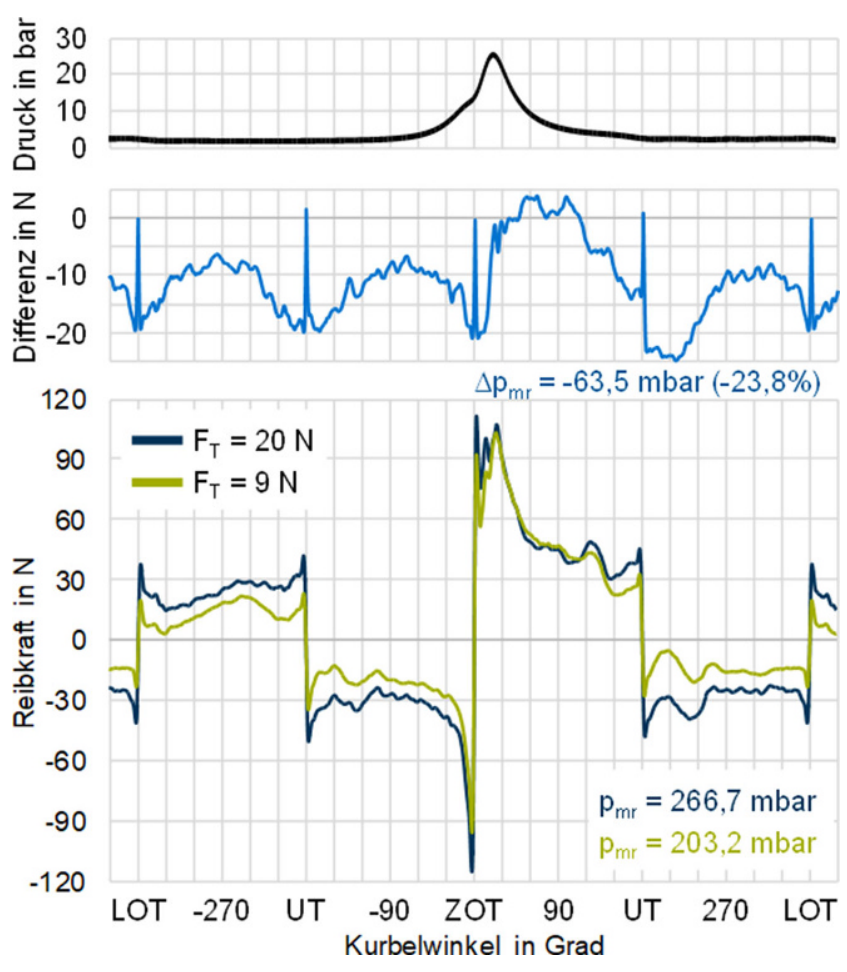

Abb. 7 Reibung der Kolbengruppe: Einfluss der Tangentialkraft des Ölabstreifrings $\left(2000 \mathrm{~min}^{-1}, 6\right.$ bar $\left.\mathrm{pmi}_{\mathrm{mi}}, \mathrm{T}_{\mathrm{Öl}}=90^{\circ} \mathrm{C}\right)$, modifiziert nach [11]

im hydrodynamischen Bereich bei hoher Kolbengeschwindigkeit wirkt. Lediglich während der Expansionsphase ist der Unterschied marginal was auf die sehr heißen Blow-By Gase zurückgeführt werden kann. Bedingt durch den hohen Brennraumdruck findet eine starke Gasströmung nach unten statt, was zu einem Temperatureintrag in den Ölfilm auf der Kolbengruppe führt. Durch die damit verbundene Viskositätsänderung nimmt die Schmierfilmdicke zwischen Ringlauffläche und Zylinderwand ab. Trotz der reduzierten Ringvorspannung tritt Dünnfilmschmierung bzw. Mischreibung ein (vgl. Abb. 1). Entscheidend für die Kraftstoffersparnis ist der Reibmitteldruck $\mathrm{p}_{\mathrm{mr}}$. Dieser kann durch die beschriebene Maßnahme am Ölabstreifring um 23,8\% gesenkt werden. Mit der Annahme, dass in diesem Betriebspunkt ca. 4 bis $8 \%$ der eingebrachten Kraftstoffenergie in der Kolbengruppenreibung verloren gehen, bedeutet dies eine $\mathrm{CO}_{2}$-Reduktion größer $1 \%$. Insbesondere mit Blick auf die geringen Kosten der Maßnahme, stellt dies eine sehr günstige Option der Effizienzsteigerung dar.

Um den Einfluss der zuvor beschriebenen heißen Brennraumgase zu vermeiden wurden weitere Tests bei drucklosem, geschlepptem Motorbetrieb durchgeführt. Des Weiteren wurde untersucht, ob sich das Potenzial mit der Öltemperatur und somit mit der Ölviskosität ändert. In Abb. 8 sind die Ergebnisse für drei verschiedene Ringspannungen $(9 \mathrm{~N}, 14 \mathrm{~N}, 20 \mathrm{~N})$, zwei Öltemperaturen $\left(35^{\circ} \mathrm{C}, 120^{\circ} \mathrm{C}\right)$ und zwei Drehzahlstufen $\left(1000 \mathrm{~min}^{-1}, 2000 \mathrm{~min}^{-1}\right)$ zusammen-

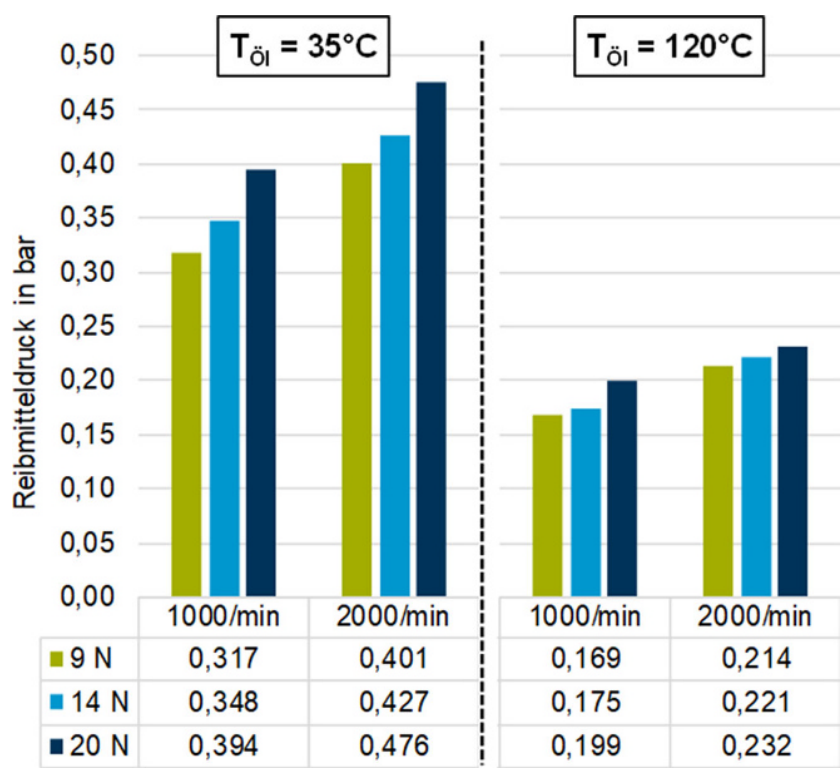

Abb. 8 Reibmitteldruckänderung über Tangentialkraftänderung Ölabstreifring (Last: 6 bar $\mathrm{p}_{\mathrm{mi}}$ ), modifiziert nach [11]

gefasst. Unabhängig von Drehzahl und Öltemperatur sinkt der Reibmitteldruck mit abnehmender Ringvorspannung. Eine Drehzahlsteigerung von 10001000 auf $2000 \mathrm{~min}^{-1}$ führt zu einer Zunahme der Reibungsverluste, womit die Theorie gemäß Stribeck (vgl. Abb. 1) bestätigt wird. Auffällig ist, dass das Potenzial der Tangentialkraftreduktion mit zunehmender Öltemperatur sinkt. Bei einer Drehzahl von $2000 \mathrm{~min}^{-1}$ und einer Öltemperatur von $35^{\circ} \mathrm{C}$ führt die Reduktion von 20 auf $9 \mathrm{~N}$ zu einem Reibleistungsrückgang von $15,8 \%$. Dieses Potenzial schrumpft bei einer Öltemperatur von $120^{\circ} \mathrm{C}$ auf $7,8 \%$. Damit werden die Beobachtungen im gefeuerten Motorbetrieb bestätigt, dass bei sinkender Ölviskosität trotz reduzierter Tangentialkraft Dünnfilmschmierung auftritt und somit der Reibungsvorteil abnimmt.

\subsection{Wirkung der reduzierten Tangentialkraft auf Ölverbrauch}

In einem zweiten Messblock wurde der Einfluss der Tangentialkraftreduktion auf die Ölverteilung innerhalb der Kolbengruppe untersucht. Um einen Laufzeiteinfluss zu vermeiden, kamen neue Ölabstreifringe gleicher Spezifikation zum Einsatz. Aufgrund von Fertigungstoleranzen unterscheidet sich der Ring mit niedriger Vorspannung geringfügig von dem Ring, der bei den Reibungsmessungen eingesetzt wurde.

Im ersten Schritt findet eine Visualisierung des Schmierfilms zwischen Kolbengruppe und Zylinderlaufbahn unter geschleppten Bedingungen statt. Im oberen Teil von Abb. 9 sind die zugehörigen LIF (Laser-induzierte Fluoreszenz) -Aufnahmen zu sehen. Unterhalb sind die abgeleiteten Schmierfilmdicken für die einzelnen Kolbenbereiche 

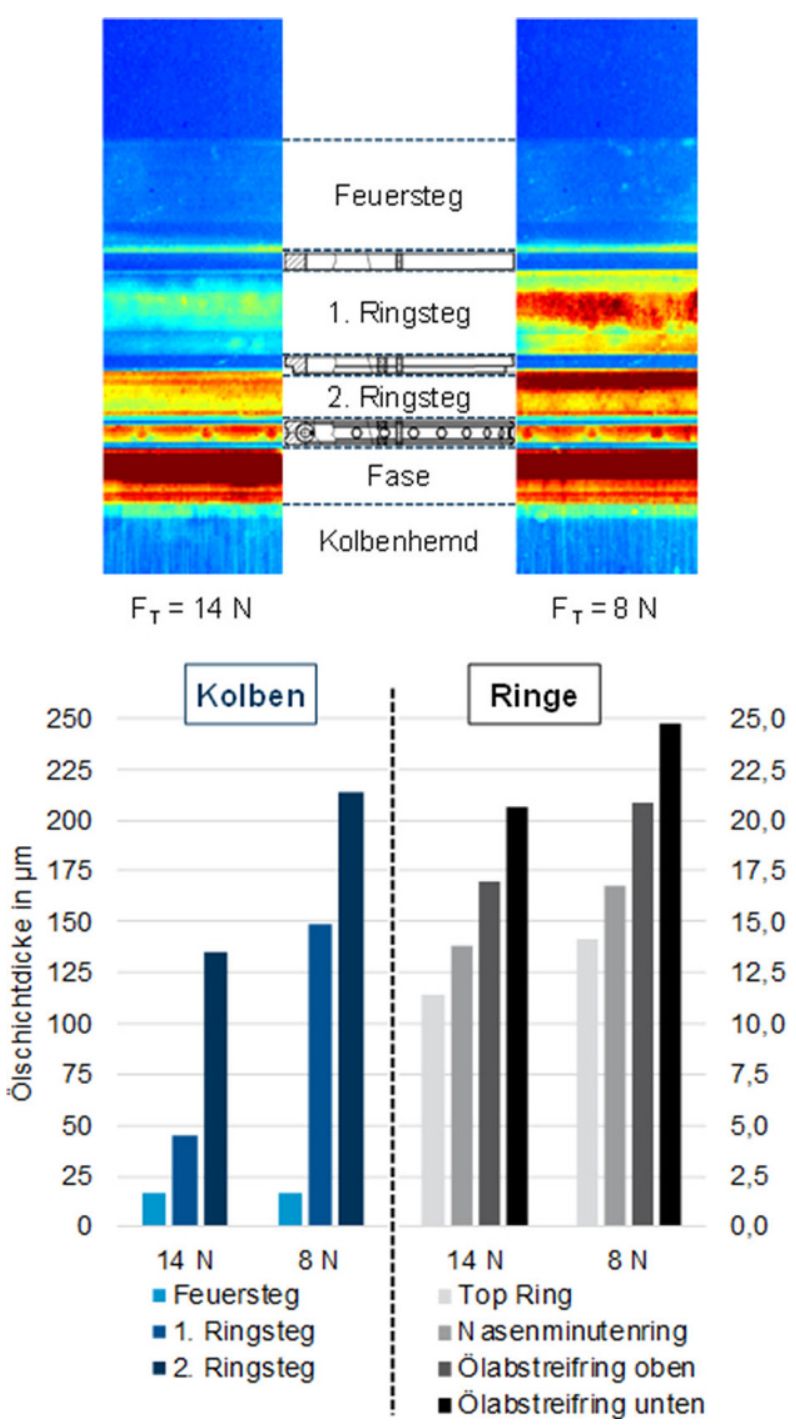

Abb. 9 Ölschichtdickenänderung über Tangentialkraftänderung Ölabstreifring (geschleppter Motor: $900 \mathrm{~min}^{-1}, 500 \mathrm{mbar} \mathrm{psau}_{\text {) }}$ )

dargestellt. Zu beachten ist, dass die Skalierung der beiden Balkendiagramme unterschiedlich gewählt ist, da die Ölschicht auf den Ringlaufflächen deutlich geringer ist als zwischen den Ringen (auf den Ringstegen).

Die Aufzeichnungen aus Abb. 9 wurden $45^{\circ} \mathrm{KW}$ nach Ladungswechsel-OT bei $900 \mathrm{~min}^{-1}$ und einem minimalen, absoluten Saugrohrdruck $p_{\text {saug }}$ von 500 mbar aufgezeichnet. Dies entspricht einer nahezu geschlossenen Drosselklappe. Der Zeitpunkt der Aufzeichnung zu Beginn der Ansaugphase wurde aus zwei Gründen so gewählt: Erstens ist das gesamte Ringfeld einsehbar und zweitens hat sich gezeigt, dass zu diesem Zeitpunkt ein vermehrtes Abschleudern von Öltröpfchen eintritt. Ein Vergleich der LIF-Bilder zeigt, dass durch Reduktion der Tangentialkraft deutlich mehr Schmieröl in die Bereiche oberhalb des Ölabstreifrings gelangt. Gerade in der Ansaugphase entsteht bei geschlossener Drosselklappe ein Unterdruck im Brennraum, der
Gasströmungen aus dem Kurbelgehäuse in Richtung Brennraum (Reverse-Blow-By) verursacht. Die Gase transportieren einen Teil des Schmieröls mit nach oben. Dabei findet die Strömung durch die Ringstöße, die Kolbenringnuten und den Kontakt Kolbenring/Zylinderlaufbahn statt. Durch die geringere Vorspannung des Ölabstreifrings kann mehr Öl den Ring/ Liner-Kontakt passieren, zudem streift der Ring weniger Öl ab und lässt somit einen größeren Anteil auf der Zylinderwand zurück. Diese Ölmenge gelangt folglich auf den 2. Ringsteg und wird weiter in Richtung 1. Ringsteg und Feuersteg transportiert. Die abgeleiteten Ölschichtdicken bestätigen den visuellen Eindruck, dass bei niedriger Tangentialkraft sowohl ein dickerer Film auf den Ringlaufflächen als auch zwischen den Ringen vorhanden ist. Jedoch dringt unter den hier gewählten Betriebsbedingungen keine größere Ölmenge zum Feuersteg vor, weshalb von keiner signifikanten Änderung des Ölverbrauchs ausgegangen werden kann.

Ein verstärkter gasgetriebener Öltransport in Richtung Brennraum wird bei höherer Drehzahl erwartet, da hier die Reverse-Blow-By Menge steigt. Die LIF-Aufnahmen aus Abb. 10 wurden bei $2700 \mathrm{~min}^{-1}$ und $500 \mathrm{mbar}_{\mathrm{Saug}}$ getätigt und zeigen eine starke Zunahme der Ölmenge in den oberen Bereichen der Kolbengruppe. Im rechten Bild (niedrige Tangentialkraft) weist der Feuersteg eine sehr hohe Signalintensität auf. Durch die gestiegenen Massenkräfte bei hoher Drehzahl löst sich das Schmieröl in Form von Tröpfchen von der Kolbenoberfläche und dringt in den Brennraum ein. Jedoch zeigen auch die Ergebnisse mit stärker vorgespanntem Ölabstreifring eine deutliche Zunahme der Ölmenge auf dem Feuersteg. Demnach wären weitere Anpassungen an der Kolbengruppe notwendig, um unter diesen Betriebsbedingungen einen Öleintrag in den Brennraum zu vermeiden.

Relevant wird dieser geschleppte Betriebszustand bei Einsatz der Motorbremse während Bergabfahrten und bei Deaktivierung einzelner Zylinder, bekannt unter dem Begriff „Zylinderabschaltung“. Aus den oben gezeigten Gründen sammelt sich während dieser Schleppzustände Motoröl im Brennraum an. Gelangen diese unverbrannten Ölpartikel in den Abgastrakt, so kommt es zu unerwünschten Ablagerungen an den Abgasnachbehandlungskomponenten. Verbleiben die Partikel im Brennraum, so können bei anschließender Aktivierung der Zündung Vorentflammungseffekte und insbesondere erhöhte Partikelemissionen entstehen. Letzteres wurde in der Studie von Gunkel et al. [3] nachgewiesen. Die aus dem Öl resultierenden Partikel wiegen mit Blick auf den zukünftigen Einsatz von eFuels noch schwerer, da diese Kraftstoffe nahezu partikelfrei verbrennen, wie in $[24,25]$ nachgewiesen. Das heißt jedoch nicht, dass die hier gezeigte reibungsreduzierende Maßnahme zwingend zu erhöhtem Ölverbrauch führen muss. Durch Anpassungen der Regelstrategie von Drosselklappe 

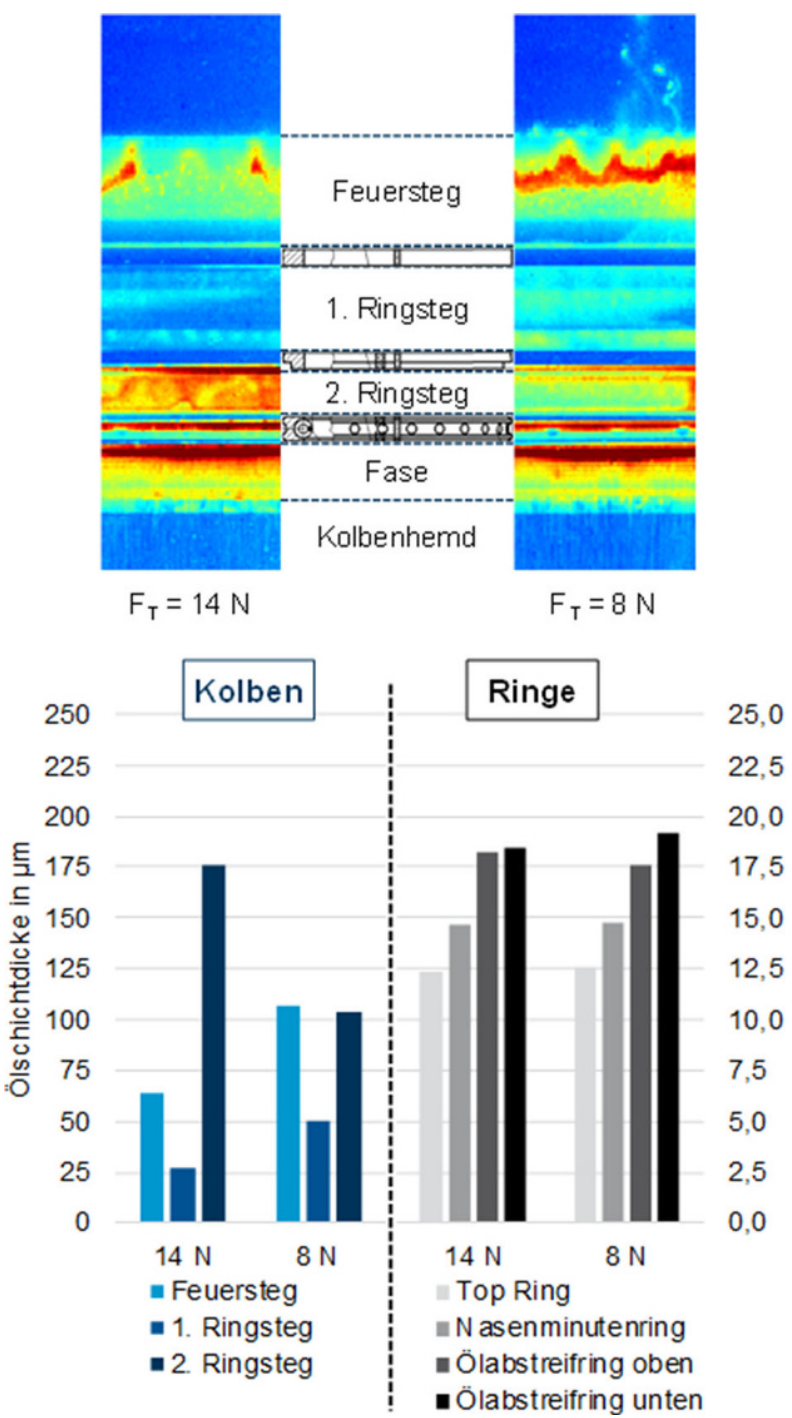

Abb. 10 Ölschichtdickenänderung über Tangentialkraftänderung Ölabstreifring (geschleppter Motor: $2700 \mathrm{~min}^{-1}, 500$ mbar $\mathrm{p}_{\text {Saug }}$ )

und Kolbenkühldüse könnte der zunehmenden Ölströmung in Richtung Brennraum entgegengewirkt werden. Des Weiteren sind bereits Konzepte zur Abkopplung einzelner Zylinder vorhanden [26]. Dies würde ein Mitschleppen der deaktivierten Zylinder verhindern.

Abschließend wurde die Maßnahme unter gefeuerten Betriebsbedingungen analysiert. Abb. 11 zeigt die Ergebnisse für die hohe Drehzahlstufe von $2700 \mathrm{~min}^{-1}$ und 6 bar indiziertem Mitteldruck. Zunächst wird deutlich, dass die Ölmenge in der gesamten Kolbengruppe geringer ist als im Schleppbetrieb. Ursächlich hierfür sind mehrere Effekte. Erstens steigt die Blow-By Menge aufgrund des höheren Zylinderdrucks. Folglich findet ein verstärkter gasgetriebener Öltransport in Richtung Kurbelgehäuse statt. Zweitens erfolgt durch die Verbrennung ein Temperatureintrag in den Ölfilm. Durch die damit verbundene Reduktion der Ölviskosität kann gemäß Stribeck vermehrt Dünn-

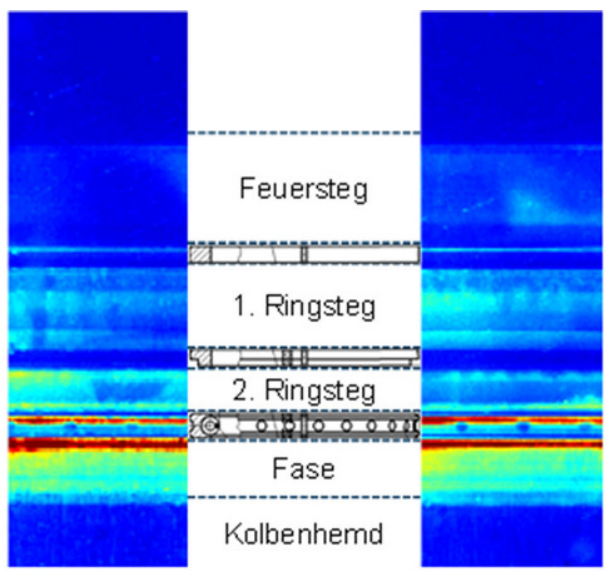

$\mathrm{F}_{\mathrm{T}}=14 \mathrm{~N}$

$F_{T}=8 \mathrm{~N}$

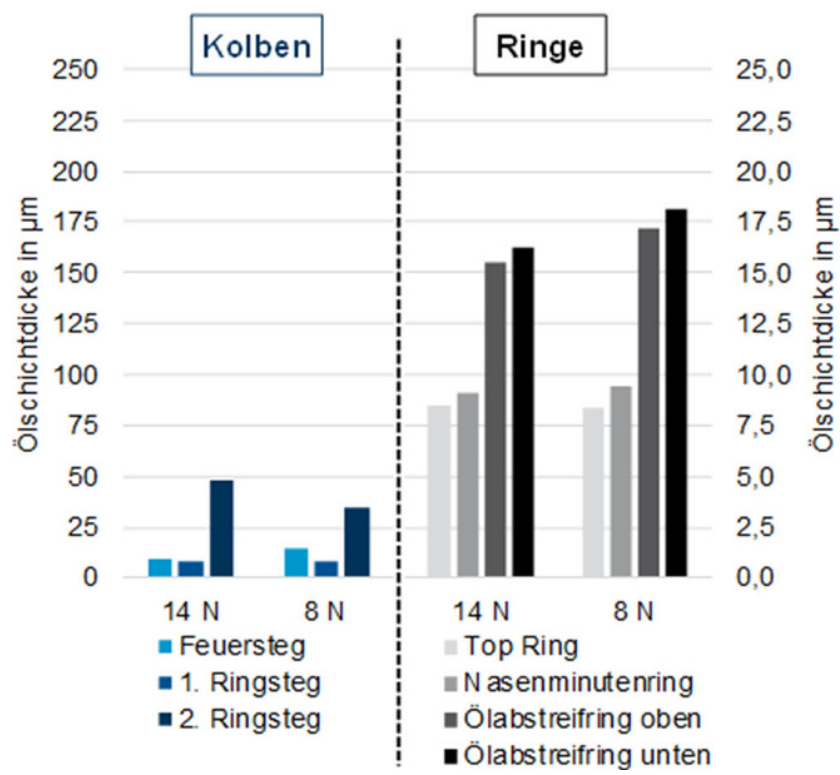

Abb. 11 Ölschichtdickenänderung über Tangentialkraftänderung Ölabstreifring (gefeuerter Motor: $2700 \mathrm{~min}^{-1}, 6$ bar $\mathrm{pmi}_{\mathrm{i}}$ )

filmschmierung eintreten. Drittens resultiert der gestiegene Brennraumdruck in einem erhöhten Anpressdruck der Kompressionsringe, was zu zunehmendem Mischreibungsanteil führt. Letzteres wird anhand der rechten Balkendiagramme deutlich. Im gefeuerten Betrieb (Abb. 11) sind die Schichtdicken an den Kompressionsringen um 4 bis $5 \mu \mathrm{m}$ geringer als im Schleppbetrieb (Abb. 10). Die Aufnahme wurde im Ansaugtakt $45^{\circ} \mathrm{KW}$ nach Ladungswechsel-OT getätigt. Es wird angenommen, dass im Bereich des ZündOTs die Schmierfilmdicke am Top Ring auf wenige Mikrometer abnimmt, verbunden mit zunehmendem Festkörperkontakt zwischen Ring und Zylinderlaufbahn. Obwohl der Ölabstreifring vom Brennraudruck nicht beeinflusst wird, sind die Schichtdicken bei gefeuertem Motor etwas geringer. Dies kann, wie bereits beschrieben, auf die niedrigere Ölviskosität zurückgeführt werden. 
Die Tangentialkraft selbst wirkt sich lediglich auf die Schmierfilmstärke an den Laufflächen des Ölabstreifrings aus. Bei niedriger Ringvorspannung ist der Film um ca. $2 \mu \mathrm{m}$ dicker als bei höherer Vorspannung. Es kann angenommen werden, dass dadurch auch etwas mehr Öl auf der Zylinderlaufbahn zurückbleibt und somit ein größerer Anteil verdampft. Da die Schichtdickendifferenzen, anders als im Schleppbetrieb, sehr gering sind, empfiehlt sich für die Zukunft der zusätzliche Einsatz einer Ölemissionsmessung mittels Time of Flight Massenspektrometer, um die genaue Auswirkung auf den Ölverbrauch zu erfassen.

Mit Blick auf Reibung und Ölverbrauch wird deutlich, dass durch Reduktion der Tangentialkraft des Ölabstreifrings ein signifikanter Reibungsvorteil erzielt werden kann. Unter gefeuerten Betriebsbedingungen führt die Maßnahme lediglich zu sehr geringem Anstieg der Ölschichtdicke, insbesondere in den oberen Bereichen der Kolbengruppe. Ein Abschleudern von Öltröpfchen tritt nicht ein, weshalb das Risiko hinsichtlich Vorentflammung, Vergiftung von Abgasnachbehandlungskomponenten und Partikelentstehung gering ist. Anders verhält es sich bei geschlepptem Motorbetrieb, der beispielsweise bei Bergabfahrt und Einsatz der Motorbremse auftritt oder während der Deaktivierung einzelner Zylinder. In diesem Fall wirkt sich eine Reduktion der Tangentialkraft negativ auf den Öleintrag in den Brennraum aus. Optimierte Regelstrategien für Drosselklappe und Kolbenkühldüse könnten unter diesen Bedingungen Abhilfe schaffen.

\section{Zusammenfassung und Ausblick}

In diesem Artikel wurde der Einfluss der Tangentialkraft des Ölabstreifrings auf die Reibungsverluste und den Ölhaushalt der Kolbengruppe eines Ottomotors bewertet. Dabei kamen zwei verschiedene Messmethoden zum Einsatz. Für die Reibungsanalyse wurde die Floating-Liner Methode angewendet, mit der eine kurbelwinkelbasierte Erfassung der Reibkraft zwischen Kolbengruppe und Zylinderlauffläche möglich ist. Die Visualisierung der Ölströmung wurde mittels einer Messtechnik basierend auf der laserinduzierten Fluoreszenz realisiert. Mit Hilfe der durchgeführten Analysen konnten folgende Erkenntnisse gewonnen werden:

- Durch Reduktion der Tangentialkraft kann ein Reibkraftvorteil im gesamten Motorzyklus erzielt werden. Da der Ölabstreifring, anders als die Kompressionsringe, kaum vom Brennraumdruck beeinflusst wird und die maßgebliche Belastung durch die Ringvorspannung erfolgt, wirkt die Maßnahme über das gesamte Arbeitsspiel.

- Der Reibleistungsvorteil einer niedrigen Tangentialkraft sinkt mit zunehmender Öltemperatur. Da mit steigender
Temperatur die Ölviskosität abnimmt, tritt trotz niedrigerer Tangentialkraft vermehrt Dünnfilmschmierung ein.

- Durch Reduktion der Ringvorspannung dringt insbesondere bei geschlepptem Motor deutlich mehr Schmieröl in die oberen Bereiche der Kolbengruppe vor. Die große Ölmenge auf dem Feuersteg kann sich teilweise von der Oberfläche lösen und in den Brennraum eindringen. Dies lässt sich darauf zurückführen, dass bei niedriger Tangentialkraft mehr Schmieröl den Kontakt Kolbenring/ Zylinderlaufbahn passieren kann.

- Unter gefeuerten Betriebsbedingungen hat die geringere Tangentialkraft keine signifikante Auswirkung auf die Ölmenge im Bereich des Feuerstegs und der Kompressionsringe. Der Betrieb unter Last führt zu einer Druckbelastung der Kompressionsringe, einem Anstieg der BlowBy Menge und einer Abnahme der Ölviskosität. Folglich nehmen die Schichtdicken im gesamten tribologischen System ab, zusätzlich erfolgt ein gasgetriebener Öltransport in Richtung Kurbelgehäuse.

Das Potenzial der reduzierten Ringvorspannung hängt unter anderem von den mechanischen und thermischen Verzügen der Laufbuchse im Betrieb ab, welche im Rahmen dieser Studie nicht erfasst wurden. Für zukünftige Untersuchungen wird empfohlen, diese Verzüge messtechnisch zu erfassen, um das Zusammenwirken von Ring und Zylinderlaufbahn besser bewerten zu können.

Im Realbetrieb wirkt sich die untersuchte Maßnahme insbesondere bei Einsatz der Motorbremse während Bergabfahrten und bei Abschaltung einzelner Zylinder negativ aus. Der damit verbundene Schleppmodus führt bei Ölabstreifringen mit niedriger Tangentialkraft zu erhöhtem Öleintrag in den Brennraum. Damit verbunden sind steigende Partikelemissionen, zunehmende Gefahr von Vorentflammung und Vergiftung von Abgasnachbehandlungskomponenten. Um das Reibungspotential dennoch nutzen zu können, wird für die Zukunft empfohlen, den Fokus auf Regelstrategien von Drosselklappe und Kolbenkühldüse zu legen. Durch Vermeidung von starkem Reverse-Blow-By bei geschlossener Drosselklappe und überflüssigem Ölangebot könnte der Öleintrag in den Brennraum auch unter diesen kritischen Randbedingungen verringert werden.

Funding The results presented were achieved in research projects sponsored by Forschungsvereinigung Verbrennungskraftmaschinen e. V. (FVV, https://www.fvv-net.de/)

Funding Open Access funding enabled and organized by Projekt DEAL.

Interessenkonflikt J. Schäffer und G. Wachtmeister geben an, dass kein Interessenkonflikt besteht.

Open Access Dieser Artikel wird unter der Creative Commons Namensnennung 4.0 International Lizenz veröffentlicht, welche die Nutzung, Vervielfältigung, Bearbeitung, Verbreitung und Wiedergabe in 
jeglichem Medium und Format erlaubt, sofern Sie den/die ursprünglichen Autor(en) und die Quelle ordnungsgemäß nennen, einen Link zur Creative Commons Lizenz beifügen und angeben, ob Änderungen vorgenommen wurden.

Die in diesem Artikel enthaltenen Bilder und sonstiges Drittmaterial unterliegen ebenfalls der genannten Creative Commons Lizenz, sofern sich aus der Abbildungslegende nichts anderes ergibt. Sofern das betreffende Material nicht unter der genannten Creative Commons Lizenz steht und die betreffende Handlung nicht nach gesetzlichen Vorschriften erlaubt ist, ist für die oben aufgeführten Weiterverwendungen des Materials die Einwilligung des jeweiligen Rechteinhabers einzuholen.

Weitere Details zur Lizenz entnehmen Sie bitte der Lizenzinformation auf http://creativecommons.org/licenses/by/4.0/deed.de.

\section{Literatur}

1. Backhaus R (2020) Paradigmenwechsel in China - Die neue Antriebsvielfalt. MTZ 81(07/08):8-15

2. Holmberg K, Andersson P, Erdemir A (2012) Global energy consumption due to friction in passenger cars. Tribol Int 47:221-234

3. Gunkel M, Frensch M, Robota A (2018) Innermotorische Emissionsreduzierung - Zusammenhang zwischen Partikelemissionen und Ölverbrauch. MTZ 79(07/08):46-50

4. An J (2014) Bestimmung der Schmierungs- und Reibungsverhältnisse im Kontakt Kolbenring gegen Zylinderlaufbahn von Verbrennungsmotoren in einem Modelltribometer. Dissertation an der Ruhr-Universität Bochum

5. Edtmayer J, Lösch S, Hick H, Walch S (2019) Comparative study on the friction behaviour of piston/bore interface technologies. Automot Engine Technol 4:101-109

6. Furuhama S, Sasaki S (1983) New device for the measurement of piston frictional forces in small engines. SAE Technical Paper 831284

7. Werner MK (2014) Entwicklung eines Motorprüfstands zur Untersuchung der Kolbengruppenreibung und deren HaupteinflussgröBen. Dissertation an der Technischen Universität München, München

8. Werner MK, Merkle A, Graf S, Holzmüller R, Wachtmeister G (2012) Calculation of the piston assembly friction: classification, validation and interpretation. SAE Technical Paper 2012-01-1323

9. Merkle A, Kunkel S, Wachtmeister G (2012) Analysis of the mixed friction in the piston assembly of a SI engine. SAE Technical Paper 2012-01-1333

10. Werner MK, Graf S, Merkle AA, Wachtmeister G (2014) Direct measurement of the piston assembly friction. MTZ Worldw 75(1):50-57

11. Merkle AA (2014) Maßnahmen zur Reduzierung der CO2-Emissionen von Verbrennungsmotoren durch Reibungsoptimierung des tribologischen Systems Kolbengruppe. Dissertation an der Technischen Universität München, München
12. Graf S, Ruch F, Mittler R (2016) Optimization of the piston assembly friction. In: ATZlive: Internationaler Motorenkongress BadenBaden

13. Kirner C, Halbhuber J, Uhlig B (2016) Experimental and simulative research advances in the piston assembly of an internal combustion engine. Tribol Int 99:159-118

14. Halbhuber J, Wachtmeister G (2020) Effect of form honing on piston assembly friction. SAE Technical Paper 2020-01-5055

15. Ito A, Chubachi Y, Yamamoto T (2015) A study on effects of low viscosity engine oil and MoDTC on piston friction losses in a DI diesel engine. SAE Technical Paper 2015-01-2044

16. Nagano Y, Ito A, Okamoto D, Yamasaka K (2019) A study on the feature of several types of floating liner devices for piston friction measurement. SAE Technical Paper 2020-01-0177

17. Thirouard B, Tian T, Hart DP (1998) Investigation of oil transport mechanisms in the piston ring pack of a single cylinder diesel engine, using two dimensional laser induced fluorescence. SAE Technical Paper 982658

18. Thirouard B (2001) Characterization and modeling of the fundamental aspects of oil transport in the piston ring pack of internal combustion engines. Dissertation am Department of Mechanical Engineering des Massachusetts Institute of Technology, Cambridge

19. Wigger S (2014) Charakterisierung von Öl- und Kraftstoffschichten in der Kolbengruppe mittels laserinduzierter Fluoreszenz. Dissertation in der Abteilung Maschinenbau und Verfahrenstechnik der Universität Duisburg-Essen, Duisburg

20. Wigger S, Müller T, Füßer H-J (2017) Tribologieanalyse der Kolbengruppe mittels optisch zugänglichem Forschungsmotor. MTZ 78(07/08):46-51

21. Schäffer J (2017) Kolbenring-Öltransport - Glasliner I. Abschlussbericht zum Vorhaben Nr. 1210. Forschungsvereinigung Verbrennungskraftmaschinen e. V., Frankfurt am Main

22. Schäffer J (2019) Kolbenring-Öltransport - Glasliner II. Abschlussbericht zum Vorhaben Nr. 1302. Forschungsvereinigung Verbrennungskraftmaschinen e. V., Frankfurt am Main

23. Schäffer J, Kirner C, Härtl M, Wachtmeister G (2019) Development of a measuring system for the visualization of the oil film between the piston and cylinder liner of a gasoline engine. SAE Technical Paper 03-13-02-0013

24. Härtl M, Stadler A, Backes F, Wachtmeister G, Jacob E (2017) Potenziell CO2-neutrale Kraftstoffe für saubere Ottomotoren. MTZ 78(07/08):80-88

25. Härtl M, Gaukel K, Pélerin D, Wachtmeister G (2017) Oxymethylenether als potenziell CO2-neutraler Kraftstoff für saubere Dieselmotoren - Teil 1: Motorenuntersuchungen. MTZ 78(02):52-59

26. Karmann SB, Rösler S, Wachtmeister G, Fischer PD, Pflaum H, Stahl K (2018) Co-simulation study of the split-crankshaft engine's electro-mechanical clutch unit. SAE Technical Paper 03-11-010006, Bd. 11, Nr. 1 